\title{
PERCEPCIÓN DE FUTUROS PROFESORES DE EDUCACIÓN FÍSICA HACIA SUS FORMADORES
}

\author{
Felipe Poblete-Valderrama
}

Universidad Santo Tomás, Sede Valdivia, Chile.Telf.: +569-99991162. Correo electrónico: felipepobleteva@santotomas.cl

\section{Luis Linzmayer Gutiérrez}

Universidad Del Bio-Bio, Sede Chillan, Chile. Telf.: +569-77654500

Correo electrónico: luislinzmayer@gmail.com

\section{Marcos García Neira}

Universidad de Sao Paulo, Brasil. Correo electrónico: mgneira@usp.br

\section{Jorge Pérez Gallardo}

Profesor Libre Docencia.Universidad de Campinas, Brasil. Telf.: +569-65726481. Correo electrónico: jsperezgallardo@gmail.com

Estimado Sr. Editor:

La percepción de los futuros docentes hacía sus formadores se torna cada vez más importante en relación al desarrollo del pensamiento crítico en los procesos de enseñanzaaprendizaje y formación docente. En este sentido, como señalan (Moreno et al. 2013), escuchar la voz del alumnado acerca de los procesos evaluativos, formativos y de enseñanza y aprendizaje vividos abre una puerta al análisis sobre la Formación de Profesores de Educación Física.

En Chile, el progreso del sistema de educación superior ha alcanzado una tendencia similar a los demás países de América Latina. Esto se ha observado en los últimos 25 años, ya que se ha producido un aumento increíble de centros de formación postsecundaria, conformándose hoy un panorama con instituciones de educación muy heterogéneas en complejidad, tamaño y oferta de carreras en el país como lo plantea Bernasconi y Rojas, (2004). En esta línea, al introducirse al sistema educativo superior y sus saberes pedagógicos, se observa que convergen una serie de aristas y conceptos inevitables de 
conjugar, como por ejemplo, en primer lugar, el desafío del profesor orientador por impregnar en sus estudiantes el deseo y la vocación por esta nueva área que es la pedagogía y todas las experiencias que esta conlleva.

Gutiérrez et al. (2007) plantean que la educación física actual tiene como objetivo la educación del alumno como un ser integral. Por otra parte, la variedad en relación al perfil del estudiante de Pedagogía en Educación Física y las motivaciones por estudiar esta disciplina, generan un sinfín de percepciones por parte de estos en relación a sus procesos de formación. De este modo, la configuración de la Educación Física moderna es el resultado de ese desarrollo, en diferentes áreas. La posibilidad de dar valor a la voz de los estudiantes en formación se torna fundamental para los procesos de mejora establecidos en las Universidades.

La percepción del estudiante se entiende como la opinión válida de éste en relación a la interacción de sus vivencias en la dinámica profesor-estudiante. (Papaioannou, 1998) afirma que la percepción ha sido investigada recabando distintas vertientes, donde esta depende de la forma en cómo el profesor desarrolla sus clases. (Friedmann, 1983) plantea interrogantes relativas a ¿qué es un buen profesor?, ¿cuál es la esencia de una buena enseñanza?, ¿qué criterios se deben seguir para evaluar la competencia de los profesores?, o ¿cuál es la forma más efectiva de producir buenos profesores?, son cuestiones de completa actualidad que se vienen planteando una y otra vez a lo largo de la historia de la educación.

En sintonía con lo anterior, la percepción de los estudiantes hacia los profesores es un tema muy común dentro de las aulas de las universidades debido a que la labor docente está supeditada a la validación de elementos como evaluación docente, evaluación de desempeño por jefaturas directas, pero además el escrutinio público de los estudiantes sobre el profesor. Frases como: un profesor es bueno, un profesor es malo, este profesor me cae bien, este profesor me cae mal, este profesor me inspira, este profesor esta muy capacitado, son solo algunos de los comentarios que se pueden encontrar en los estudiantes en formación. Dichas manifestaciones surgen desde la relación y la forma en que este profesor se relaciona, aborda y transfiere los contenidos a los estudiantes, siendo en esta dinámica donde se entrelazan las percepciones de los estudiantes hacia sus profesores. Como resultado de esta interrelación, se creará un relación positiva o negativa, muy rara vez 
neutra como lo expone Arón y Milicic (2009), siendo difícil que un profesor sea percibido como alguien afectivamente neutral por sus estudiantes. De manera regular se registran evaluaciones docentes por parte de la universidad en relación al desempeño del docente con instrumentos de Encuesta de Evaluación Docente (resultados que en contadas ocasiones son conocidos por los estudiantes, sino más bien recolectados y guardados por la universidad para la mejora de sus procesos), siendo instrumentos impuestos y ya formulados por la universidad en áreas que son de su interés.

No obstante, la percepción de los estudiantes hacia sus profesores obedece a un cúmulo de aspectos para la configuración de esta, dentro de estas dimensiones no solo se perciben aspectos teóricos y/o disciplinares en donde el profesor debe demostrar conocimiento, sino que también se consideran aspectos transversales como empatía, trato y consideraciones frente a situaciones emergentes. Olmedo y Peinado (2008) muestran que los aprendices desean no sólo un buen trato por parte del profesor, sino también exposiciones claras, el respeto y la puntualidad.

Dentro del proceso de mejora docente en muy pocas instancias se considera la percepción de los estudiantes, entendiendo que estos poseen una opinión contextualizada, siendo por tanto válida y digna de consideración y análisis. Pérez y Alfaro (1997) estiman que si los estudiantes son los destinatarios de la educación, son ellos los que mejor pueden valorarla, a pesar de que tengan una visión parcial, su opinión proporcionará un referente significativo que hay que considerar. Dentro de este orden de ideas, uno de los aspectos menos considerados a la hora de reorientar las prácticas docentes es la percepción de los estudiantes hacia sus docentes lo que coincide con lo planteado por Gutiérrez (2003) quien manifiesta que un aspecto insuficientemente considerado en todo este proceso ha sido la valoración que los alumnos hacen de sus profesores. Según Cárcamo (2012) la percepción hacia los profesores varía según la estructura de la personalidad de cada individuo, pero también dependerá del resultado de la calidad de la interacción que haya experimentado el alumno con el profesor.

La preparación del docente en una didáctica para el cambio, es el reto de los académicos universitarios (Mazón et al. 2009) y para ello el profesor no tiene por qué ser un simple dictador o instructor de clases; como lo expresa Cásares (2000) debe ser ante 
todo, un líder, gran educador y formador, su misión es dirigir el proceso de educación de la personalidad y aprendizaje de los estudiantes, abarcando las dimensiones cognitivas y afectivas, sociales y culturales, por eso es tan importante su labor y considerar como los estudiantes lo valoran o perciben, de esta forma mejorar las prácticas y contribuir en el proceso de enseñanza aprendizaje. Finalmente se hace necesario establecer de manera clara los aspectos teleológicos que busca entregar y desarrollar la institución formadora y profesores en los estudiantes, permitirá el fortalecimiento del profesor de educación física en contexto escolar dejando en claro cual es la función y/o rol de este en el contexto escolar fortalecimiento de la Formación de la Ciudadanía Soberana.

\section{Referencias Bibliográficas}

Aron, A. \& Milicic, N. (2009). Clima social escolar y desarrollo personal. Santiago: Andrés Bello.

Bernasconi, A., \& Rojas, F. (2004) Informe sobre la educación superior en Chile: 19802003. Santiago (Chile): Editorial Universitaria.

Cárcamo, J. (2012). El profesor de educación física desde la perspectiva de los escolares. Estudios Pedagógicos, 38, 1, 105-119.

Cásares, D. (2000). Lideres y educadores. El maestro, creador de una nueva sociedad. México: Universidad del Valle de México.

Gutiérrez, M. (2003). Manual sobre valores en la educación física y el deporte. Barcelona: Paidós.

Gutiérrez, S., Pilsa, D. \& Torres, B. (2007). Perfil de la educación física y sus profesores desde el punto de vista de los alumnos. Revista Internacional de Ciencias de Deporte, 3, 39-52.

Mazón, J., Martínez, J. \& Martínez, A. (2009). La evaluación de la función docente mediante la opinión del estudiante. Un nuevo instrumento para nuevas dimensiones: COED. Revista de la Educación Superior, 28,149, 13-139.

Moreno, A. Trigueros, C. \& Rivera, E. (2013). Autoevaluación y emociones en la formación inicial de profesores de educación física. Estudios Pedagógicos, 34, 1, 
165-177.

Olmedo, K. \& Peinado, S. (2008). El perfil del profesor universitario. Desde la perspectiva de los estudiantes. Primer congreso Internacional sobre profesorado principiante e inserción profesional a la docencia. Sevilla, España.

Papaioannou, A. (1998). Students' perceptions of the physical education class environment for boys and girls and the perceived motivational climate. Research Quarterly for exercise and sport, 69, 267-275.

Pérez, A. \& Alfaro, I. (1997) La evaluación de la docencia en la Universidad de Valencia. España. Congreso Pedagogía. La Habana. 